\title{
Single Myofiber Isolation and Culture from a Murine Model of Emery-Dreifuss Muscular Dystrophy in Early Post-Natal Development
}

\author{
Gloria Pegoli ${ }^{1,2}$, Federica Lucini ${ }^{1,2}$, Chiara Mozzetta $^{3}$, Chiara Lanzuolo ${ }^{1,2,4}$ \\ ${ }^{1}$ Istituto Nazionale di Genetica Molecolare "Romeo ed Enrica Invernizzi" ${ }^{2}$ IRCCS Santa Lucia Foundation ${ }^{3}$ Department of Biology and Biotechnology, "C. \\ Darwin" Sapienza University, CNR - IBPM ${ }^{4}$ CNR - Institute of Biomedical Technologies (ITB)
}

\section{Corresponding Authors}

Chiara Mozzetta

chiara.mozzetta@uniroma1.it

Chiara Lanzuolo

chiara.lanzuolo@cnr.it

\section{Citation}

Pegoli, G., Lucini, F., Mozzetta, C., Lanzuolo, C. Single Myofiber Isolation and Culture from a Murine Model of Emery-Dreifuss Muscular Dystrophy in Early Post-Natal Development. J. Vis. Exp. (161), e61516, doi:10.3791/61516 (2020).

\section{Date Published}

July 1,2020

DOI

$10.3791 / 61516$

URL

jove.com/video/61516

\section{Abstract}

Autosomal dominant Emery-Dreifuss muscular dystrophy (EDMD) is caused by mutations in the LMNA gene, which encodes the A-type nuclear lamins, intermediate filament proteins that sustain the nuclear envelope and the components of the nucleoplasm. We recently reported that muscle wasting in EDMD can be ascribed to intrinsic epigenetic dysfunctions affecting muscle (satellite) stem cells regenerative capacity. Isolation and culture of single myofibers is one of the most physiological exvivo approaches to monitor satellite cells behavior within their niche, as they remain between the basal lamina surrounding the fiber and the sarcolemma. Therefore, it represents an invaluable experimental paradigm to study satellite cells from a variety of murine models. Here, we describe a re-adapted method to isolate intact and viable single myofibers from post-natal hindlimb muscles (Tibialis Anterior, Extensor Digitorum Longus, Gastrocnemius and Soleus). Following this protocol, we were able to study satellite cells from Lamin $\Delta 8-11$-/- mice, a severe EDMD murine model, at only 19 days after birth.

We detail the isolation procedure, as well as the culture conditions for obtaining a good amount of myofibers and their associated satellite-cells-derived progeny. When cultured in growth-factors rich medium, satellite cells derived from wild type mice activate, proliferate, and eventually differentiate or undergo self-renewal. In homozygous Lamin $\Delta 8-11$-/- mutant mice these capabilities are severely impaired.

This technique, if strictly followed, allows to study all processes linked to the myofiberassociated satellite cell even in early post-natal developmental stages and in fragile muscles. 


\section{Introduction}

Skeletal muscle is a differentiated tissue with one of the most extended ability to regenerate after exercise or trauma ${ }^{1}$. This characteristic is mainly due to the presence of stem cells, called satellite cells because of their peripheral position between the basal lamina and the plasmalemma of the myofiber $^{2}$. During post-natal development, satellite cells proliferate and progressively differentiate, thus contributing to skeletal muscle growth. Once in the adulthood, satellite cells enter a reversible quiescent state, and upon physiological or pathological trauma, they activate, proliferate and differentiate in order to repair the damaged muscles ${ }^{3}$. Defects in the capacity of satellite cells to properly transit through these different regenerative phases and to undergo self-renewal have been firmly linked to muscle wasting, either during physiological aging ${ }^{4,5,6}$ or in muscle degenerative diseases, such as muscular dystrophies $7,8,9,10$.

Two main culture approaches exist to study satellite cells ex-vivo: primary myogenic cultures from mononucleated cells, mechanically and chemically dissociated from whole muscle 11,12 ; or culture of isolated myofibers ${ }^{13}, 14,15,16,17,18,19,20$. In the first case, the process of satellite cells isolation involves the trituration of whole muscles extracted from the mouse, a chemical digestion, filtration and fluorescent activated cell sorting $(\text { FACS })^{21}$. This procedure, although effective in isolating satellite cells from a variety of models, entails several variables that expose satellite cells to stress and disrupts their physiological niche 22,23 . By contrast, myofiber isolation involves a gentler digestion of muscle tissue with matrix degrading enzymes and a mechanical shredding that causes reduced trauma to stem cells ${ }^{20}$. This second approach allows a much more efficient retrieval of viable satellite cells, that remain physically attached to their myofiber between the basal lamina and the sarcolemma, thus allowing analysis within their physiological niche ${ }^{19,20}$.

Many different protocols have been proposed during the past years to properly and efficiently isolate single myofibers from skeletal muscles. Already in 1986 Bischoff proposed a protocol to isolate fibers from the Flexor Digitorum Brevis ${ }^{13}$ and later, in 1995, Rosenblatt et al. modified the protocol to obtain a more efficient separation of myofibers ${ }^{14}$. Since then, many other authors proposed adjusted procedures on other muscles, such as Extensor Digitorum Longus (EDL) and Tibialis Anterior (TA $)^{15}, 16,17,18,19,20$, that are longer, even if more fragile, muscles ${ }^{14}$. Isolated myofibers can then be cultivated both in adhesion, to allow for the expansion of satellite cells-derived myoblasts, or in floating conditions, up to 96 hours, to follow the progeny derived from single satellite cells ${ }^{19}$ (Figure 1). Variable concentrations of serum within the culture medium are used to trigger satellite cells activation, proliferation and/or differentiation, to study their capacity to properly transit through these different phases ${ }^{1}$.

We recently described the epigenetic mechanism behind the exhaustion of the satellite stem cell pool in the mouse model of EDMD, the Lamin $\Delta 8-11$-/- mouse ${ }^{7}$. Since these mice usually die between 4-8 weeks of age ${ }^{24}$, due to severe muscle loss, an attempt was made to capture the molecular defects underlying the early onset of the disease by focusing our analysis on post-natal muscle development. Floating single myofibers were isolated and cultured from wild type and Lamin $\Delta 8-11$-/- mutant ${ }^{7} 19$ days-old mice. At this stage, muscle defects are already evident, but mice are still viable. However, since all the above-mentioned protocols for single 
myofibers extraction were optimized for skeletal muscles of adult mice, we needed to adapt them to our purposes: very small mice in term of age and size, and very fragile myofibers. Thus, we describe here our re-adaptation of the protocol proposed by the Rudnicki laboratory ${ }^{19}$ to obtain a significant number of single viable myofibers from mice during post-natal development and from severe dystrophic muscles, such as those derived from Lamin $\Delta 8-11$-/- mice ${ }^{24}$. The final goal of this approach is to provide a standardized procedure for the study of myofibers-associated muscle stem cells in any other mouse model when the early stages of postnatal development are of interest, or in the case of mouse models carrying any specific disease that makes myofibers more susceptible to mechanical stress.

\section{Protocol}

All the experimental procedures were performed under the ethical approval of the Italian Ministry of Health and the Institutional Animal Care and Use Committee (authorization n. 83/2019-PR). The animals were maintained in an authorized facility at San Raffaele Hospital, Milan, Italy (authorization n. N. 127/2012-A).

\section{Muscle dissection and myofiber culture}

1. Equipment preparation.

1. Before starting, prepare all the necessary solutions as described in Table 1. These solutions need to be freshly prepared.

2. Clean all the surfaces and tools that will be used during the procedure with $70 \%$ ethanol.

3. Before starting with mice sacrifice, perform coating of $100 \mathrm{~mm}$ and $35 \mathrm{~mm}$ Petri dishes using Horse Serum (HS). Coat all dishes to prevent myofibers from attaching to the plastic. Consider using one $100 \mathrm{~mm}$ dish and four $35 \mathrm{~mm}$ dishes per mouse.

4. After removing the excess of HS, store coated dishes in an incubator at $37{ }^{\circ} \mathrm{C}$ for $30 \mathrm{~min}$. Then fill it with washing solution or culture medium (two $35 \mathrm{~mm}$ dishes per mouse).

NOTE: Alternatively, a solution of $10 \% \mathrm{HS}$ in Dulbecco's modified Eagle's medium (DMEM) can be used to coat dishes. Always use coated dishes. It is possible to use culture dishes of different size, but small dimension Petri dishes are recommended.

5. For fibers isolation, prepare sterile Pasteur pipettes as shown in Figure 2. For each mouse prepare one large hole bore pipette for muscle handling and mechanical disaggregation (Figure 2A) and one small hole pipette for fibers selection (Figure 2B). Cut each glass pipette, possibly using a diamond pen, to the desired length and smooth pipette's edges on a flame.

6. Coat each pipette by briefly wetting it with HS before use.

2. Mouse sacrifice and muscle dissection

1. Pre-warm the digestion solution at room temperature for 10 min before starting the dissection procedure. Polypropylene FACS round-bottom tubes are the most suitable containers for the purpose.

2. Immediately before the beginning of muscle retrieval, sacrifice the mouse according to proper national IACUC recommendation.

3. Wet its lower body with $70 \%$ ethanol before cutting the skin to make the removal of the hair easier. 
4. Put the mouse in a prone position on a support of polystyrene covered with aluminum paper and cut the skin starting from the middle of the back longitudinally and in the direction of the legs.

5. Carefully remove the skin without touching the muscles and tendons. It is possible to rip off all the skin.

6. Cut the two legs of the mouse and rapidly proceed with the dissection.

NOTE: If it is more comfortable, it is possible to continue the dissection on the entire mouse but working on the leg allows more mobility and precision in the later cuts.

7. Fix the leg on the support at the level of the foot using a pin and start isolating skeletal muscles of interest in this order: TA, EDL, Gastrocnemius and Soleus.

8. Lift the lower tendon of TA with a sharp tweezer at ankle height and cut it, then cut with fine scissor all around the TA muscle to the other tendon at the level of the patella (Figure 3A). Transfer into the digestion solution.

9. Lift the lower tendon of EDL and separate it from other muscles by gently pulling it upward up to the other tendon. Cut and place it into the digestion solution.

NOTE: Since EDL may be extremely small to be cut separately from TA, they can be dissected together (Figure 3B). Then, if the entire muscle is too big, cut it in 2-3 pieces starting from the tendon and following the fibers in a longitudinal direction (Figure 3C).

10. Rotate the leg showing the back muscles and fix the foot using the pin. Lift the Achille's tendon, Gastrocnemius will automatically separate from other muscles. The upper tendon is up in the back of the patella. Cut it and add the muscle to digestion.

11. Lift the external tendon of the leg (with respect to the body) and obtain the Soleus. Gently separate it from the other muscles by scrolling under with the tweezer.

12. Do the same for the other leg (steps from 1.2.7. to 1.2.11.).

3. Muscle digestion

1. Incubate the digestion solution containing all the muscles in a water bath at $37{ }^{\circ} \mathrm{C}$ for about $45-50$ min. During the digestion time, regularly check the muscle to avoid over-digestion. Every 10 min invert the digestion tubes $10 x$ with an energetic movement (avoid vortexing).

2. Stop the digestion when muscles start to loosen up and myofibers are visible.

3. At the very end of the digestion time shake the samples.

4. To stop the digestion, carefully transfer the digestion suspension to a pre-warmed $100 \mathrm{~mm}$ Petri dish with $10 \mathrm{~mL}$ of washing solution.

NOTE: Avoid muscle over-digestion as this will inevitably result in the isolation of hyper-contracted myofibers. Usually with this protocol, muscles of homozygous mutant mice take $45 \mathrm{~min}$ to be digested, while muscles of wild type mice take 50-55 min. Digestion time needs to be experimentally validated.

4. Single myofibers isolation

1. First isolate the fibers already dissociated under a dissecting microscope by picking them individually with a coated P200 pipette or small hole Pasteur and 
transfer them from the $100 \mathrm{~mm}$ Petri into a new $35 \mathrm{~mm}$

Petri dish with $5 \mathrm{~mL}$ of pre-warmed washing solution.

2. To release further myofibers, pipette the muscle up and down using a large hole bore glass pipette with warm medium, until fibers are mechanically released.

Do not be too persistent as this will result in damaging fibers.

3. Continue releasing myofibers from the muscle until the dish contains a desirable amount. If the Petri dish is kept at room temperature for more than $8 \mathrm{~min}$, stop and perform a minimum of 5 min incubation at $37^{\circ} \mathrm{C}$, $5 \% \mathrm{CO}_{2}$ to re-equilibrate the medium.

4. Before transferring the single myofibers to the culture medium, leave them at $37{ }^{\circ} \mathrm{C}, 5 \% \mathrm{CO}_{2}$ in wash dish for at least $1 \mathrm{~h}$. This helps myofibers to adjust to the in vitro condition.

NOTE: Adult myofibers are less susceptible to stress and can be washed $\sim 2-3 x$. However, in this condition (at 19 days post-natal age) it is better to perform only one washing step to prevent myofiber damage. Therefore, always pay attention to keep selected myofibers sufficiently clean by not carrying debris or hypercontracted fibers.

5. Single myofiber culture

1. Transfer individual myofibers to a new pre-warmed dish with the appropriate culture medium (high serum medium to allow satellite cells activation, see Figure 1).

2. Change the medium to the isolated myofibers, by transferring them to a new coated dish with new culture medium, only after $48-72 \mathrm{~h}$ of culture to avoid any stress that will lead to myofibers hypercontraction and disruption.

\section{Downstream applications: Myofibers crosslinking and immunofluorescence}

NOTE: The myofibers-associated satellite cells can be visualized by immunofluorescence (IF) at the time of interest. Since most of the published protocols are optimized to perform IF on adult myofibers, here a detailed protocol is presented to obtain reliable results also on myofibers isolated from post-natal muscles.

1. Myofiber crosslinking

1. Before starting, prepare all the necessary solutions as described in Table 2.

2. Precoat with $\mathrm{HS}$ as many $1.5 \mathrm{~mL}$ microcentrifuge tubes as the number of samples. Be sure to remove all HS before proceeding. Since crosslinked fibers are tougher than the living ones and more difficult to pipette, be sure to crosslink about 200-300 fibers separately per tube.

3. Under a dissecting microscope, collect all the fibers that can be considered healthy, transfer them to the microcentrifuge tube and leave the tube vertical for 5 min inside the incubator to allow fibers to settle.

4. Remove the supernatant very slowly from the tube.

5. Crosslink fibers by adding $1 \mathrm{~mL}$ of $4 \%$ paraformaldehyde (PFA) at RT to the tube. Do it gently to avoid fibers distress.

6. To prevent fibers interweaving during the crosslinking, keep the tube in very gentle agitation for $10 \mathrm{~min}$.

7. Keep the tube in a vertical position for 5 min at RT to allow fibers to settle, then discard the supernatant ensuring to remove the majority of PFA volume. 
8. Add $1 \mathrm{~mL}$ of phosphate buffered saline (PBS) and keep the tubes vertical for $5 \mathrm{~min}$ at RT to let the fibers to sediment.

9. Remove the supernatant and repeat the washing procedure (step 2.1.8.) twice again.

10. Keep crosslinked samples at $4{ }^{\circ} \mathrm{C}$.

NOTE: It is possible to keep crosslinked myofibers at $4{ }^{\circ} \mathrm{C}$ for a week. If the fibers remain more than a week in this condition, this will inevitably result in myofibers intertwinement.

2. Immunofluorescence

1. Keep the tubes containing the fibers standing at RT for at least $5 \mathrm{~min}$ to allow for fiber sedimentation.

2. Remove the supernatant, leaving just a small volume to be sure not to remove any fiber.

3. Add $1 \mathrm{~mL}$ of $0.5 \%$ Triton $\mathrm{X}-100$ in PBS and incubate for 5 min with gentle agitation.

4. Put tubes in vertical position for $5 \mathrm{~min}$, then remove supernatant.

5. Add $1.5 \mathrm{~mL}$ of PBS and incubate with gentle agitation for $5 \mathrm{~min}$.

6. Keep tubes in vertical position for $5 \mathrm{~min}$, then remove the supernatant.

7. Add $1 \mathrm{~mL}$ of blocking solution and incubate for $1 \mathrm{~h}$ at RT with gentle agitation.

8. Keep tubes $5 \mathrm{~min}$ in vertical position, then remove the supernatant.

9. Dilute primary antibodies in blocking solution, add them to the tubes and incubate over-night at $4{ }^{\circ} \mathrm{C}$ in gentle agitation (for suggested concentrations see
NOTE: Alternatively, the primary antibody can be incubated for $3 \mathrm{~h}$ at RT. However, overnight incubation gave optimal staining. The incubation volume for both primary and secondary antibodies should be of $300 \mu \mathrm{L}$ when sedimented fibers reach the $100 \mu \mathrm{L}$ notch of the $1.5 \mathrm{~mL}$ microcentrifuge tube. When fibers are less abundant, a volume of $100-200$ $\mu \mathrm{L}$ is recommended.

10. Leave the tubes in vertical position for $5 \mathrm{~min}$ and then remove the supernatant.

11. Perform 3 washes in $1 \mathrm{~mL}$ of $0.25 \%$ Tween-20 in PBS, incubating for $5 \mathrm{~min}$ in gentle agitation and then leaving the tubes standing for 5 min each time.

NOTE: From here on, perform all the steps in the dark, to avoid fluorochromes bleaching.

12. Dilute secondary antibodies in blocking solution, add them to the tubes and incubate for $1 \mathrm{~h}$ at RT with gentle agitation (for concentrations see Table of Materials).

13. Wash twice in $1 \mathrm{~mL}$ of $0.1 \%$ Tween-20 in PBS, incubating for $5 \mathrm{~min}$ with gentle agitation and then leaving the tubes in standing position for $5 \mathrm{~min}$.

14. Remove the supernatant, add $1 \mathrm{~mL}$ of DAPI solution and incubate for 5 min with gentle agitation.

15. Let the tubes stand vertically in a rack for $5 \mathrm{~min}$, then remove the supernatant.

16. Wash with $1 \mathrm{~mL}$ of PBS, incubating for $5 \mathrm{~min}$ with gentle agitation and then leaving the tubes stand in a rack for $5 \mathrm{~min}$.

17. Remove the supernatant, leaving a volume of about $50 \mu \mathrm{L}$.

Table of Materials). 
3. Mounting of fluorescently labeled myofibers on microscope slides

1. Cut a P200 pipette tip and coat it with blocking solution NOTE: To coat the tip, pipette the solution up and down several times before picking the fibers, this will avoid fibers to stick to the tip wall.

2. Collect the fibers from the tube and spread them on a microscope glass slide.

3. Under a dissecting microscope, using only the natural light reflected by the mirror, use a new (not cut) P200 pipette tip to spread the fibers and to remove the excess liquid solution.

4. Leave the slides to air dry in the dark for about $10-15$ min, until very low amount of solution remains.

5. Add the mounting medium on the slide (the proper amount of mounting medium should be calibrated on the dimension of the coverslip: for a $24 \times 40 \mathrm{~mm}$ coverslip, $20 \mu \mathrm{L}$ is enough) and then slowly lay a cover glass on the area containing the fibers. Be careful not to create bubbles between the glasses.

6. Press the coverslip so that the fibers will lay on a single horizontal plan.

7. Fix the coverslip using nail polish.

8. Store the samples at $4{ }^{\circ} \mathrm{C}$ for up to 4 weeks.

9. Acquire the desired images of fluorescently labeled myofibers with a confocal microscope.

\section{Representative Results}

We typically digest four different muscles (TA, EDL, Soleus and Gastrocnemius) to retrieve a good amount of long and viable fibers that could survive $96 \mathrm{~h}$ in growth-factors rich medium (Figure 4A,B). Only the most intact fibers should be transferred in culture medium, as they will survive; all the others, that are easy to discriminate and select, need to be discarded.

When myofibers are maintained in a growth-factors rich medium satellite cells derived from wild type mice start to activate and proliferate, see Figure 1. Upon $48 \mathrm{~h}$ of culture, in healthy condition (Lamin $\Delta 8-11+/+$ ), satellite cells upregulate MyoD and undergo their first division. Activated Pax7+/MyoD+ satellite cells then proliferate and by $72 \mathrm{~h}$ in culture, they generate cell aggregates bound to the myofiber, that are even more visible at $96 \mathrm{~h}$ (Figure 5). During these divisions, some of them can repress MyoD expression, undergoing self-renewal to repopulate the stem cells pool, while those that maintain MyoD become committed to differentiation by downregulating Pax7 expression. After $96 \mathrm{~h}$, satellite cells clusters contain clearly visible MyoG+ committed cells, that can differentiate into new myofibers (Figure 1 and Figure 6). Notably, with this experiment, we described a delayed dynamics of satellite cell differentiation in homozygous mutant Lamin $\Delta 8-11$ mice (-/-) as compared to their wild type counterparts $(+/+)$, see Figure 6.

The final outcome of each single experiment let us think that the protocol developed for single myofibers isolation and culture from this model of severe muscle dystrophy ensures good quality myofibers for all further applications. 


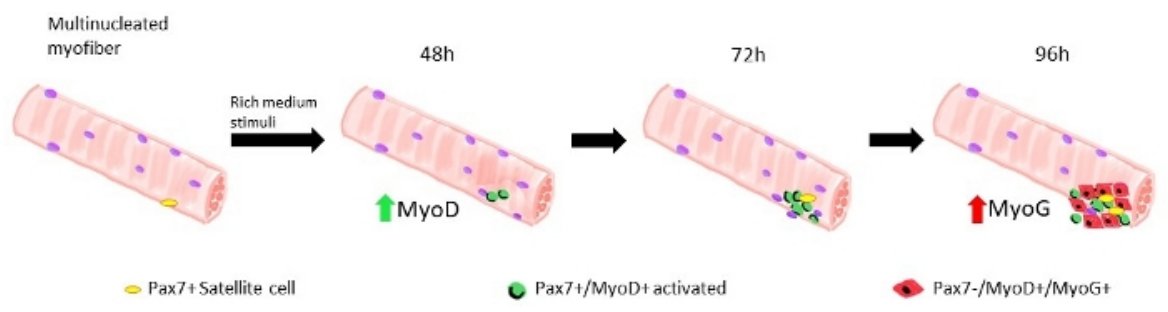

Figure 1: Graphical representation of satellite cells' regenerative phases modelled in floating myofibers. Upon 48 $\mathrm{h}$ of culture in growth-factors rich medium, Pax7+ cells get activated and undergo the first division, giving rise to a doublet of Pax7+/MyoD+ cells. MyoD positive cells then proliferate and expand, giving rise, in $72 \mathrm{~h}$ of culture, to a cluster of several cells which are the progeny of a single satellite cell. Upon $96 \mathrm{~h}$ of culture Pax7+/MyoD+ cells become differentiating Pax7-/ MyoG+ cells. During the expansion phase, a subset of Pax7+/MyoD+ cells downregulate MyoD expression undergoing selfrenewal into quiescence. Please click here to view a larger version of this figure.

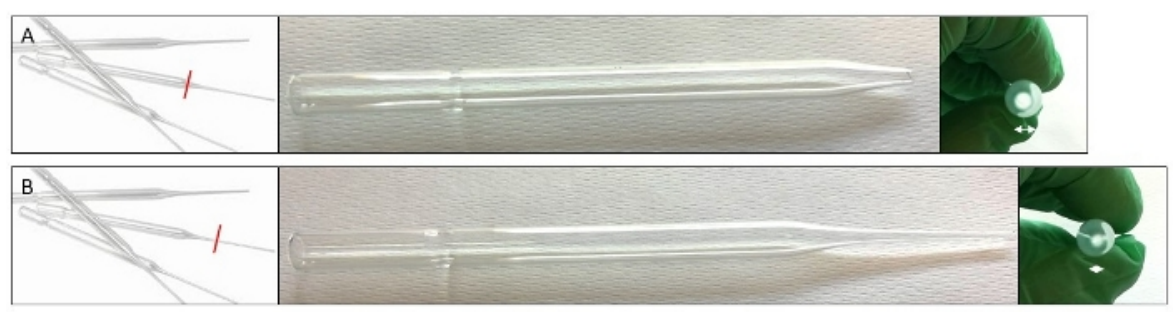

Figure 2: Preparation of bore Pasteur pipettes. (A) Longitudinal and frontal view of how the big hole bore pipette must appear. (B) Longitudinal and frontal view of how the small hole bore pipette must finally appear. Please click here to view a larger version of this figure. 

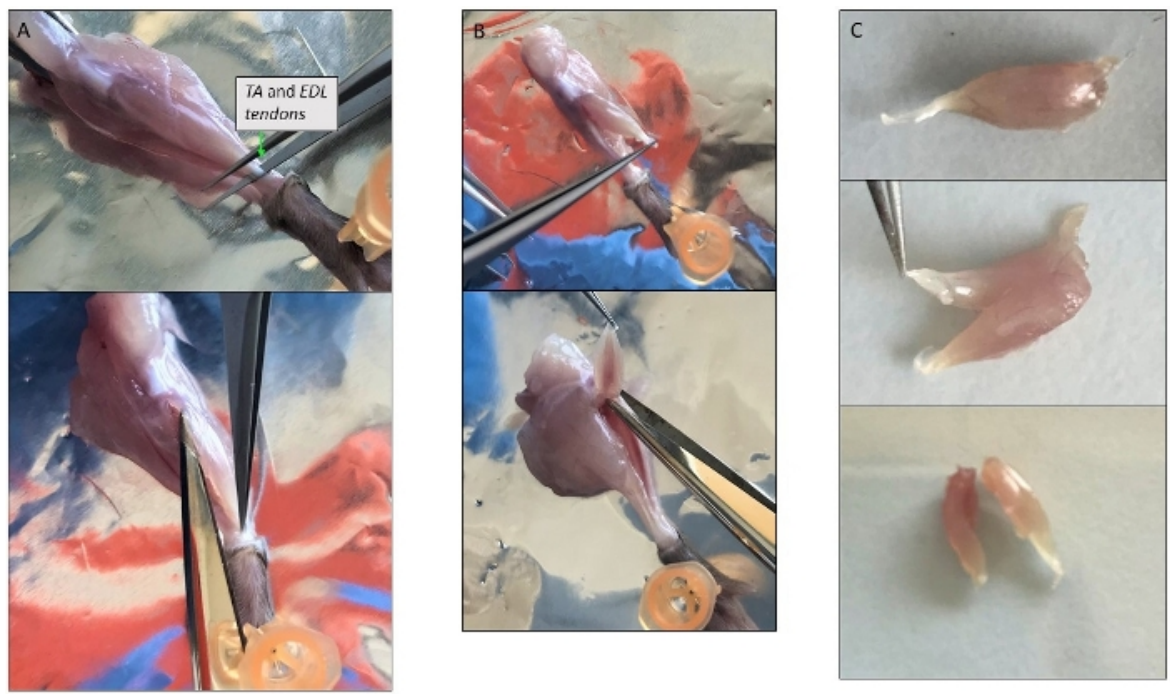

Figure 3: Representative pictures of single muscle dissection. (A) Isolation of the TA muscle. Avoiding the removal of the thin layer covering the muscle protects the myofibers inside. (B) TA and EDL muscles isolated together still attached to their upper tendon at the level of the patella. (C) Division of TA and EDL after isolation by cutting them along the longitudinal axis. Please click here to view a larger version of this figure.
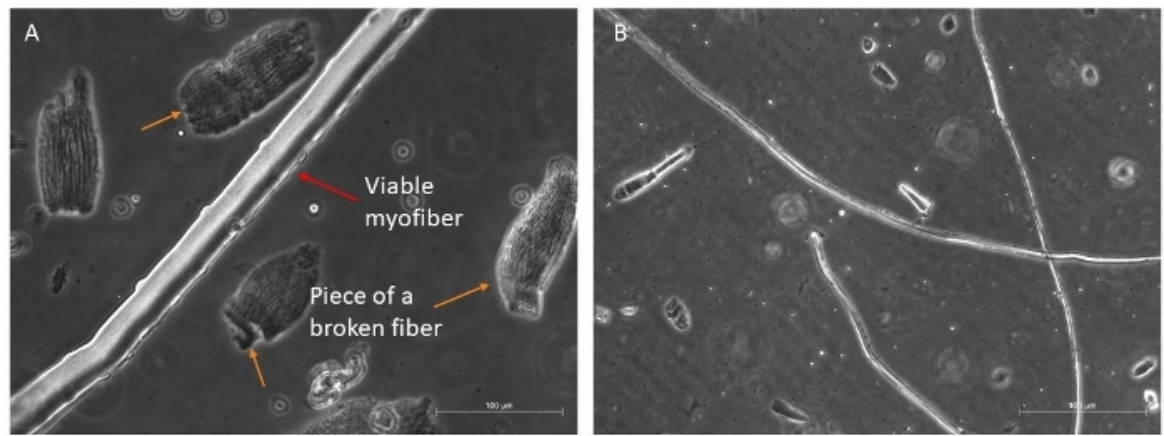

Figure 4: Examples of healthy and viable myofibers. Representative phase contrast images of viable myofibers in suspension. (A) The red arrow indicates a myofiber with visible sarcomere organization and a satellite cell on its side; the orange arrows indicate some pieces of broken myofibers, and some debris present in the first dish before the final selection for culture. Scale bar $100 \mu \mathrm{m}$. (B) More complete view of other myofibers under a smaller magnification. Scale bar $500 \mu \mathrm{m}$. Please click here to view a larger version of this figure. 

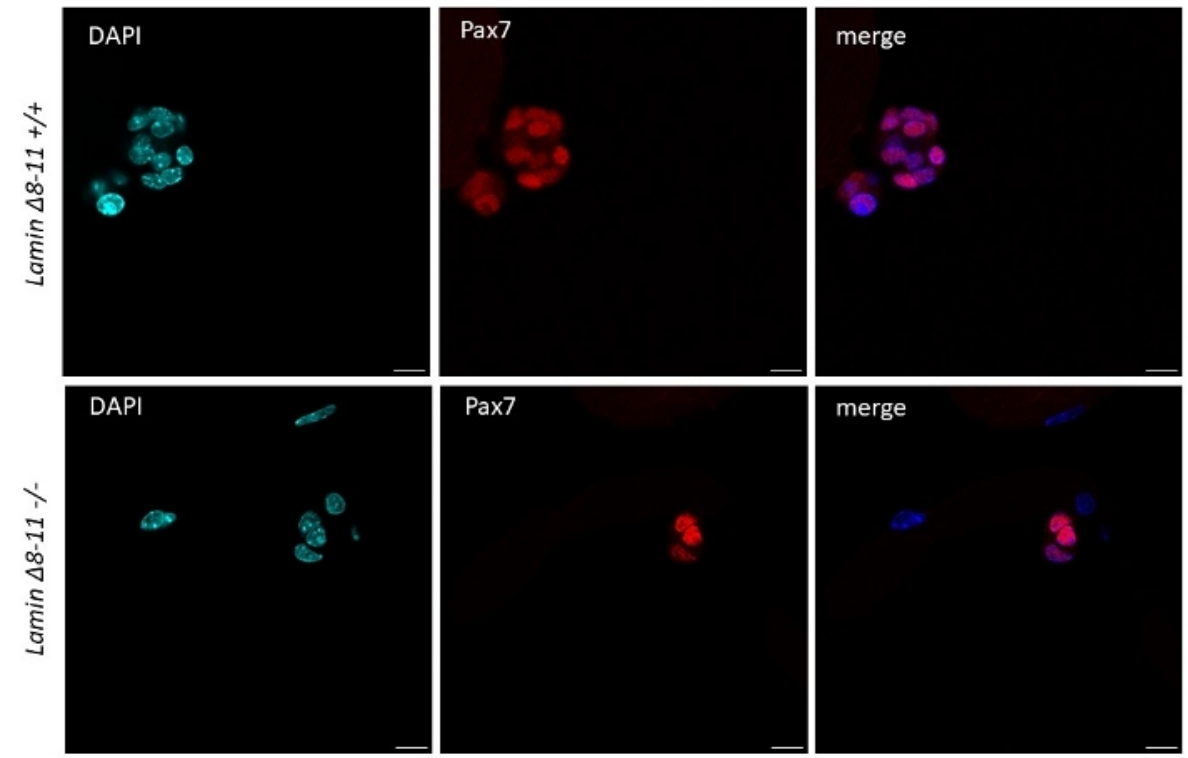

Figure 5: Difference in the dimension of stem cell clusters in wt and mutant mice. Immunofluorescence staining of myofibers extracted from 19 days Lamin $\Delta 8-11$ mice (+/+ and -/-) after $96 \mathrm{~h}$ of culture. Pax7+ satellite cells are shown. The dimension of the cell cluster in most of the cases was significantly bigger in Lamin $\Delta 8-11+/+$ than in Lamin $\Delta 8-11-/-$ in terms of number of cells. Scale bar $10 \mu \mathrm{m}$. Please click here to view a larger version of this figure.

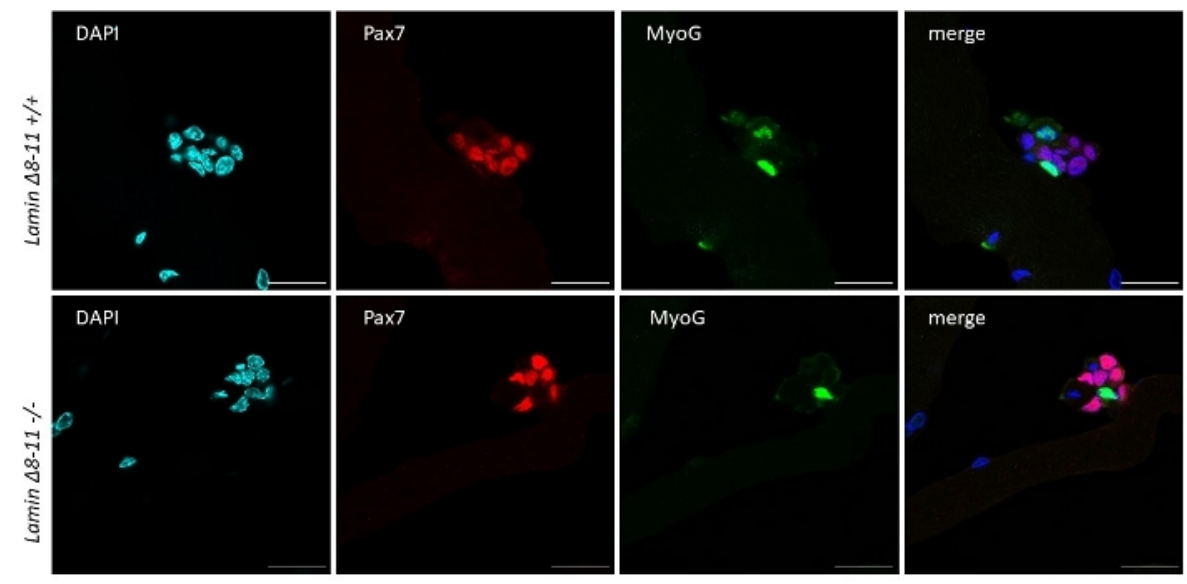

Figure 6: Representative immunofluorescence experiment. Immunofluorescence experiment performed, after $96 \mathrm{~h}$ of culture, on myofibers extracted from 19 days Lamin $\Delta 8-11$ mice (+/+ and -/-). Pax7+/MyoG- (red) and Pax7-/MyoG+ (green) cells were observed. Images obtained with a confocal microscope. Scale bar $25 \mu \mathrm{m}$. Please click here to view a larger version of this figure. 


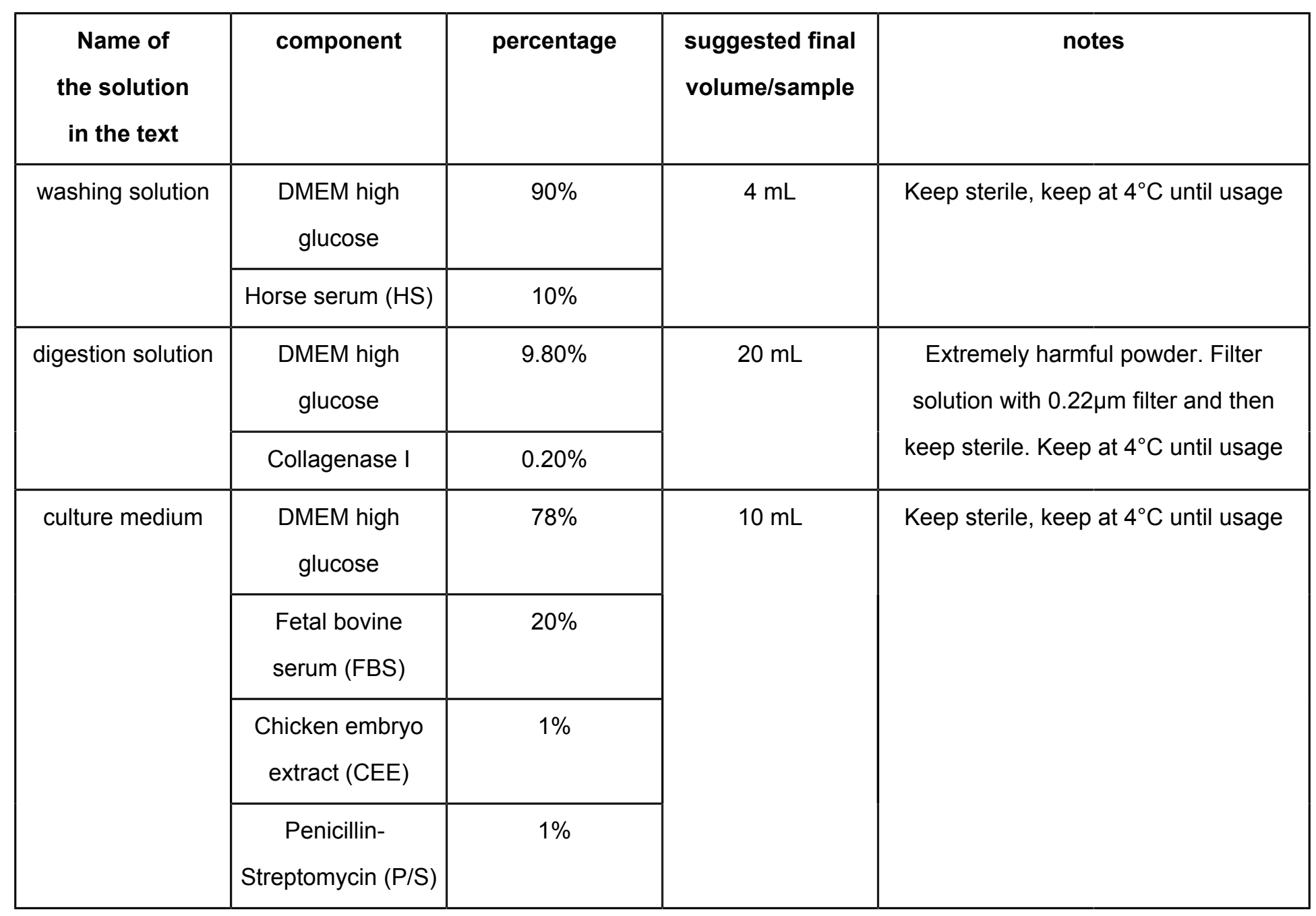

Table 1: Recipes for solutions used in section 1.

Copyright $\odot 2020$ JoVE Creative Commons Attribution-NonCommercial-NoDerivs 3.0 Unsorted

jove.com

July $2020 \cdot 161 \cdot$ e61516 Page 11 of 15

License 


\begin{tabular}{|c|c|c|c|c|}
\hline $\begin{array}{l}\text { Name of } \\
\text { the solution } \\
\text { in the text }\end{array}$ & component & percentage & $\begin{array}{l}\text { suggested final } \\
\text { volume/sample }\end{array}$ & notes \\
\hline \multirow[t]{2}{*}{$4 \%$ PFA } & $\begin{array}{l}\text { Paraformaldehyde } \\
\text { (PFA) }\end{array}$ & $4 \%$ & \multirow[t]{2}{*}{$2-3 \mathrm{~mL}$} & \multirow[t]{2}{*}{$\begin{array}{l}\text { Powder and then solution } \\
\text { are extremely harmful }\end{array}$} \\
\hline & PBS & $96 \%$ & & \\
\hline \multirow[t]{2}{*}{$0.5 \%$ Triton $\mathrm{X}-100$} & Triton $\mathrm{X}-100$ & $0.50 \%$ & \multirow[t]{2}{*}{$2-3 \mathrm{~mL}$} & \multirow{2}{*}{$\begin{array}{l}\text { Triton } \mathrm{X}-100 \text { is extremely } \\
\text { viscous, preferentially cut the } \\
\text { tip of the pipette to aliquot it }\end{array}$} \\
\hline & PBS & $99.50 \%$ & & \\
\hline \multirow[t]{2}{*}{$0.25 \%$ Tween-20 } & Tween-20 & $0.25 \%$ & \multirow[t]{2}{*}{$10 \mathrm{~mL}$} & \multirow{2}{*}{$\begin{array}{l}\text { Tween- } 20 \text { is extremely } \\
\text { viscous, preferentially cut the } \\
\text { tip of the pipette to aliquot it }\end{array}$} \\
\hline & PBS & $99.75 \%$ & & \\
\hline \multirow[t]{2}{*}{$0.1 \%$ Tween-20 } & Tween-20 & $0.10 \%$ & \multirow[t]{2}{*}{$10 \mathrm{~mL}$} & \multirow{2}{*}{$\begin{array}{l}\text { Tween- } 20 \text { is extremely } \\
\text { viscous, preferentially cut the } \\
\text { tip of the pipette to aliquot it }\end{array}$} \\
\hline & PBS & $99.90 \%$ & & \\
\hline \multirow[t]{2}{*}{ blocking solution } & $\begin{array}{l}\text { Fetal bovine } \\
\text { serum (FBS) }\end{array}$ & $10 \%$ & \multirow[t]{2}{*}{$10 \mathrm{~mL}$} & \multirow{2}{*}{$\begin{array}{c}\text { Prepare fresh solution and store } \\
\text { at } 4^{\circ} \mathrm{C} \text { for no longer than } 3 \text { weeks } \\
\text { (always check clearness before usage) }\end{array}$} \\
\hline & PBS & $90 \%$ & & \\
\hline \multirow[t]{2}{*}{ DAPI } & DAPI & $0.10 \%$ & \multirow[t]{2}{*}{ 2-3 mL } & \multirow[t]{2}{*}{ Keep in the dark } \\
\hline & PBS & $99.90 \%$ & & \\
\hline
\end{tabular}

Table 2: Recipes for solutions used in section 2.

\section{Discussion}

Isolation of intact single myofibers is an essential method in the field of myogenesis when the main objective is to characterize cell-autonomous regenerative capacities of muscle stem cells within their niche, in healthy and pathological conditions. However, when biochemical or genomic studies are of interest, FACS-isolated satellite cells might be the best option.
Single myofibers isolation allows to follow ex-vivo, but in the most physiological way, the dynamics of all the steps single satellite cells undergo during muscle regeneration, that are: activation, cell division (asymmetric and symmetric), differentiation and return to quiescence by self-renewal. Once myofibers are grown in floating conditions, the single satellite cells activate and expand forming a cluster of cells, all deriving from the same satellite cell. Immunofluorescence analysis for 
proliferation, differentiation, activation or stemness markers is then optimal to quantify the proportion between cell stages.

The key step in our protocol to obtain viable and intact myofibers can be considered the rapid but gentle muscle dissection, by tendon-to-tendon isolation, to avoid any muscle damage. Our advice is to use only sharp scissors and small sharp tweezers and to limit the entire muscle dissection procedure to ten minutes. When it is difficult to isolate very small muscles (i.e., EDL and TA), it is possible to cut them together and to later divide them by using fine scissors cutting along the longitudinal plan following the fibers. This strategy will eventually give less intact myofibers, but viability will not be compromised. The same must be performed on big muscles like Gastrocnemius to facilitate digestion. Optimization of digestion time, which needs to be empirically validated, and minimal manipulation of isolated fibers are also two crucial aspects for the positive outcome of subsequent analysis.

The advantage of the protocol reported here is that it can be applied on very small mice (in age and dimension), even when their muscles are extremely fragile. Even if not mentioned above, it is possible to follow this protocol of dissection to then culture viable myofibers for longer period using basement membrane-coated dishes ${ }^{18,19}$. It is important to consider that this situation is completely different from floating condition, where adhesion stimuli and proximity stimuli are absent.

\section{Disclosures}

No competing interests.

\section{Acknowledgments}

We thank Andrea Bianchi, the Italian Network of Laminopathies and the members of the laboratory for the support and all the constructive comments. We are grateful to Chiara Cordiglieri for the precious help at confocal microscope. The authors thank Dr. Beatrice Biferali for her help in taking pictures for figures. The work presented in here was supported by My First AIRC Grant n. 18535, AFM-Telethon n. 21030, the Italian Minister of Health n. GR-2013-02355413 and Cariplo 2017-0649 to C.L. C.M. is supported by My First AIRC grant n.18993 and AFM-Telethon n. 22489.

\section{References}

1. Wang, Y. X., Dumont, N. A., Rudnicki, M. A. Muscle stem cells at a glance. Journal of Cell Science. 127 (21), 4543$4548(2014)$.

2. Le Grand, F., Rudnicki, M. A. Skeletal muscle satellite cells and adult myogenesis. Current Opinion in Cell Biology. 19 (6), 628-633 (2007).

3. Zammit, P. S., Partridge, T. A., Yablonka-Reuveni, Z. The Skeletal Muscle Satellite Cell: The Stem Cell That Came in From the Cold. Journal of Histochemistry \& Cytochemistry. 54 (11), 1177-1191 (2006).

4. Franco, I. et al. Somatic mutagenesis in satellite cells associates with human skeletal muscle aging. Nature Communications. 9 (1), 800 (2018).

5. Cosgrove, B. D. et al. Rejuvenation of the muscle stem cell population restores strength to injured aged muscles. Nature Medicine. 20 (3), 255-264 (2014).

6. Bernet, J. D. et al. p38 MAPK signaling underlies a cellautonomous loss of stem cell self-renewal in aged skeletal muscle. Nature Medicine. 20 (3), 265-271 (2014). 
7. Bianchi, A. et al. Dysfunctional polycomb transcriptional repression contributes to lamin $\mathrm{A} / \mathrm{C}$-dependent muscular dystrophy. Journal of Clinical Investigation. 130(5):2408-2421 (2020).

8. Blau, H. M., Webster, C., Pavlath, G. K. Defective myoblasts identified in Duchenne muscular dystrophy. Proceedings of the National Academy of Sciences. 80 (15), 4856-4860 (1983).

9. Jiang, C. et al. Notch signaling deficiency underlies age-dependent depletion of satellite cells in muscular dystrophy. Disease Models \& Mechanisms. 7 (8), 9971004 (2014).

10. Vallejo, D. et al. PITX2 Enhances the Regenerative Potential of Dystrophic Skeletal Muscle Stem Cells. Stem Cell Reports. 10 (4), 1398-1411 (2018).

11. Danoviz, M. E., Yablonka-Reuveni, Z. Skeletal Muscle Satellite Cells: Background and Methods for Isolation and Analysis in a Primary Culture System. Methods in Molecular Biology. (798), 21-52 (2012).

12. Sacco, A., Doyonnas, R., Kraft, P., Vitorovic, S., Blau, H. M. Self-renewal and expansion of single transplanted muscle stem cells. Nature. 456 (7221), 502-506 (2008).

13. Bischoff, R. Proliferation of muscle satellite cells on intact myofibers in culture. Developmental Biology. 115 (1), 129-139 (1986).

14. Rosenblatt, J. D., Lunt, A. I., Parry, D. J., Partridge, T. A. Culturing satellite cells from living single muscle fiber explants. In Vitro Cellular \& Developmental Biology Animal. 31 (10), 773-779 (1995).

15. Shefer, G., Yablonka-Reuveni, Z. Isolation and Culture of Skeletal Muscle Myofibers as a Means to Analyze
Satellite Cells. Methods in Molecular Biology. (290), 281304 (2005).

16. Anderson, J. E., Wozniak, A. C., Mizunoya, W. Single Muscle-Fiber Isolation and Culture for Cellular, Molecular, Pharmacological, and Evolutionary Studies. Methods in Molecular Biology. 798, 85-102 (2012).

17. Verma, M., Asakura, A. Efficient Single Muscle Fiber Isolation from Alcohol-Fixed Adult Muscle following $\beta$-Galactosidase Staining for Satellite Cell Detection. Journal of Histochemistry \& Cytochemistry. 59 (1), 60-67 (2011).

18. Keire, P., Shearer, A., Shefer, G., Yablonka-Reuveni, Z. Isolation and Culture of Skeletal Muscle Myofibers as a Means to Analyze Satellite Cells. Methods in Molecular Biology. 946 (206), 431-468 (2013).

19. Pasut, A., Jones, A. E., Rudnicki, M. A. Isolation and Culture of Individual Myofibers and their Satellite Cells from Adult Skeletal Muscle. Journal of Visualized Experiments. 73, e50074 (2013).

20. Gallot, Y., Hindi, S., Mann, A., Kumar, A. Isolation, Culture, and Staining of Single Myofibers. Bio-Protocol. 6 (19), 1942 (2016).

21. Liu, L., Cheung, T. H., Charville, G. W., Rando, T. A. Isolation of skeletal muscle stem cells by fluorescenceactivated cell sorting. Nature Protocols. 10 (10), 16121624 (2015).

22. Machado, L. et al. In Situ Fixation Redefines Quiescence and Early Activation of Skeletal Muscle Stem Cells. Cell Reports. 21 (7), 1982-1993 (2017).

23. Lucini, F., Bianchi, A., Lanzuolo, C. Formaldehydemediated snapshot of nuclear architecture. Methods in Molecular Biology. in press (2020). 
24. Sullivan, T. et al. Loss of a-Type Lamin Expression Compromises Nuclear Envelope Integrity Leading to Muscular Dystrophy. The Journal of Cell Biology. 147 (5), 913-920 (1999). 